\title{
THE NULL SET OF THE FOURIER TRANSFORM FOR A SURFACE CARRIED MEASURE
}

\author{
LIMIN SUN
}

(Communicated by J. Marshall Ash)

\begin{abstract}
Let $d u$ be a smooth positive measure carried by a smooth compact hypersurface $S$ that is strictly convex and without boundary in $R^{n} \quad(n \geq 2)$. Assume that both $S$ and $d u$ are symmetric about the origin. If $\hat{d} u$ denotes the Fourier transform of $d u$ then we show that the null set of $\hat{d} u$ is a disjoint union of a compact set and countably many hypersurfaces that are all diffeomorphic to the unit sphere $S^{n-1}$.
\end{abstract}

\section{INTRODUCTION}

The Fourier transforms of surface carried measures play an important role in many research areas related to harmonic analysis such as the restriction theorems and the maximal averages over hypersurfaces (cf. [2] and the literature cited therein). It turns out that the geometric property of the hypersurface is crucial to those investigations.

For a smooth measure $d u$ on some smooth hypersurfaces $s$ in $R^{n}$, the Fourier transform of $d u$ is defined by

$$
\hat{d} u(y)=\int_{S} e^{-i x \cdot y} d u(x), \quad y \in R^{n} .
$$

Let $N(S, d u)=\left\{y \in R^{n} ; \hat{d} u(y)=0\right\}$ be the null set of $\hat{d} u$. If $d \sigma$ is the standard surface measure on the unit sphere $S^{n-1}$, then

$$
\hat{d} \sigma(y)=(2 \pi)^{n / 2} J_{(n-2) / 2}(|y|) \cdot|y|^{-(n-2) / 2} .
$$

Hence, the null set of $\hat{d} \sigma$ consists of countably many hyperspheres in $R^{n}$, i.e.,

$$
N\left(S^{n-1}, d \sigma\right)=\bigcup_{j=1}^{\infty}\left\{y \in R^{n} ;|y|=r_{j}\right\} \quad \text { (disjoint union), }
$$

where $r_{j}(j=1,2, \ldots)$ is the enumeration of the positive zeros of the Bessel function $J_{(n-2) / 2}(r)$.

From the geometric point of view, it is natural to ask what happens to $N(S, d u)$ if $d u$ is a general smooth positive measure on some smooth hypersurface $S$ with nonzero Gaussian curvature.

Received by the editors September 12, 1991 and, in revised form, November 27, 1991.

1991 Mathematics Subject Classification. Primary 42B25.

Key words and phrases. Surface carried measure, Fourier transform, zero set. 
In this paper, the smooth hypersurface $S$ is assumed to be strictly convex (i.e., with positive Gaussian curvature) and without boundary. Assume further that both $S$ and $d u$ are symmetric about the origin (i.e., $x \in S$ implies $-x \in$ $S$, and $d u(E)=d u(-E)$ for any measurable set $E \subset S)$. The main result is as follows.

Theorem. Let $d u$ be a smooth positive measure carried by a smooth compact hypersurface $S$ in $R^{n}(n \geq 2)$. Suppose that $S$ and $d u$ satisfy the assumptions stated above. Then $N(S, d u)=N_{0} \cup\left(\bigcup_{j=1}^{\infty} N_{j}\right)$ (disjoint union), where $N_{0}$ is a compact set and $N_{j}(j=1,2, \ldots)$ is diffeomorphic to the unit sphere $S^{n-1}$.

\section{Preliminaries}

In order to prove the theorem, we should understand the principal part of $\hat{d} u$ as well as that of the derivative of $\hat{d} u$ in radial direction. For this purpose, we need an exact formula of $\hat{d} u$. For $y \in R^{n}$, write $r=|y|$ and $\theta=y /|y|$. The following result is due to Greenleaf [1].

Lemma 1. Let $S$ and $d u$ be given as in the theorem. Then

$$
\hat{d} u(y)=C_{n} \cdot d(\theta)^{n-2} \cdot\left[\left(t^{-(n-2) / 2} J_{(n-2) / 2}(t)\right) * \hat{g}(\theta, t)\right](r d(\theta)),
$$

where $C_{n}$ is a constant, $d(\theta) \in C^{\infty}\left(S^{n-1}\right), g(\theta, s) \in C^{\infty}\left(S^{n-1} \times R\right)$, and $g(\theta, s) \in C_{0}^{\infty}(R)$ for each fixed $\theta \in S^{n-1} ; \hat{g}(\theta, t)$ is the Fourier transform of $g(\theta, s)$ about the variable $s ; *$ denotes the convolution about the variable $t$.

Remark 1. Lemma 1 is a particular case of Lemma 6 in [1], which deals with general smooth measures (without the restriction of positiveness and symmetricity).

Proposition 1. Let $d(\theta)$ and $g(\theta, s)$ be as in Lemma 1. Then $d(\theta)>0$ for all $\theta \in S^{n-1}$ and $g(\theta, s) \neq 0$ for all $(\theta, s) \in S^{n-1} \times[-1,1]$.

Proof. From the proof of Lemma 6 in [1], it is obvious that $d(\theta)>0$. Moreover, $g(\theta, s)$ is an extension of the function $\tilde{f}(\theta ; d(\theta) s)$. In fact, for $(\theta, s) \in$ $S^{n-1} \times[-1,1]$,

$$
g(\theta, s)=\tilde{f}(\theta ; d(\theta) s)=\int_{S^{n-2}} f\left(\theta ; d(\theta) s, x^{\prime}\right)^{-1} \frac{d u}{d v} d \theta\left(x^{\prime}\right)
$$

where $d v$ is the induced Lebesgue measure on $S, d \sigma\left(x^{\prime}\right)$ is the standard surface measure on $S^{n-2}$, and $f$ is smooth and nonzero (cf. [1, pp. 529530]). Since we assume that $d u$ is positive, the three measures that appeared in (1) are all positive. Thus, the conclusion follows.

Proposition 2. The value of $g(\theta, s)$ can be modified such that $g(\theta, s)$ is an even function of $s$ and $g(\theta, s)=0$ for all $\theta \in S^{n-1}$ and $|s| \geq 2$. Meanwhile, the conclusion of Lemma 1 remains true.

Proof. Again from the proof of Lemma 6 in [1], one easily sees that the central symmetricity of $S$ and $d u$ ensures that $\tilde{f}(\theta ; d(\theta) s)$ is an even function of $s$. Moreover, for each fixed $\theta \in S^{n-1}, g(\theta, s)$ is an arbitrary extension of $\tilde{f}(\theta ; d(\theta) s) \quad(s \in[-1,1])$ to an element of $C_{0}^{\infty}(R)$. Hence, we can easily make $g(\theta, s)$ be even about $s$. Choose an even function $k(s) \in C_{0}^{\infty}(R)$ such 
that $k(s)=1$ for $s \in[-1,1]$ and $k(s)=0$ for $|s| \geq 2$. Then the conclusion of Lemma 1 remains true when $g(\theta, s)$ is replaced by $g(\theta, s) k(s)$. This completes the proof of Proposition 2.

Let $\mathscr{S}(R)$ be the Schwartz class of rapidly decreasing functions on $R$.

Lemma 2. Suppose that $h(t) \in \mathscr{S}(R)$ is an even function. For $p \geq q \geq 0$, let $H(r)=\left[\left(t^{-q} J_{p}(t)\right) * h(t)\right](r)$, where $J_{p}$ is the pth Bessel function. Then there exists a constant $C_{1}$ independent of $r$ such that

$$
H(r)=\left(\frac{2}{\pi}\right)^{1 / 2} \cdot r^{-(q+1 / 2)}\left[\hat{h}(1) \cos \left(r-\frac{1}{2} \pi p-\frac{1}{4} \pi\right)+R_{1}(r)\right]
$$

and $\left|R_{1}(r)\right| \leq C_{1} r^{-1}$ for $r>1$, where $\hat{h}$ is the Fourier transform of $h$. Proof. Since $h(t) \in \mathscr{S}(R), C_{q, h}=\operatorname{Sup}_{t \in R}|h(t)| \cdot|t|^{(q+3)}<\infty$. Then (2) $\int_{|t|>r / 2}|h(t)| d t \leq C_{q, h} \cdot \int_{|t|>r / 2}|t|^{-(q+3)} d t \leq C_{q} \cdot C_{q, h} \cdot r^{-(q+2)}$ for $r>0$ and

$$
\begin{aligned}
\left(\int_{|t|>r / 2}|h(t)|^{4 / 3} d t\right)^{3 / 4} & \leq C_{q, h}\left(\int_{|t|>r / 2}|t|^{-4(q+3) / 3} d t\right)^{3 / 4} \\
& \leq C_{q}^{\prime} \cdot C_{q, h} \cdot r^{-(q+9 / 4)} \\
& \leq C_{q}^{\prime} \cdot C_{q, h} \cdot r^{-(q+3 / 2)} \text { for } r>1 .
\end{aligned}
$$

Write $a_{p}=\frac{1}{2} \pi p+\frac{1}{4} \pi$. Since $h(t)$ is even, $\hat{h}(1)=\int_{-\infty}^{\infty} h(t) \cos t d t$. Thus we have

$$
\left\{\begin{array}{l}
H(r)-\left(\frac{2}{\pi}\right)^{1 / 2} \cdot \hat{h}(1) r^{-(q+1 / 2)} \cos \left(r-a_{p}\right)=I_{1}+I_{2}+I_{3}, \\
I_{1}=\int_{|t| \leq r / 2}\left[(r-t)^{-q} \cdot J_{p}(r-t)\right. \\
\left.\quad-\left(\frac{2}{\pi}\right)^{1 / 2} r^{-(q+1 / 2)} \cos \left(r-a_{p}\right) \cos t\right] h(t) d t, \\
I_{2}=\int_{|t|>r / 2}(r-t)^{-q} J_{p}(t) h(t) d t, \\
I_{3}=-\left(\frac{2}{\pi}\right)^{1 / 2} r^{-(q+1 / 2)} \cos \left(r-a_{p}\right) \cdot \int_{|t|>r / 2} h(t) \cos t d t
\end{array}\right.
$$

It follows from (2) that for $r>1$,

$$
\begin{aligned}
\left|I_{3}\right| & \leq\left(\frac{2}{\pi}\right)^{1 / 2} r^{-(q+1 / 2)} \int_{|t|>r / 2}|h(t)| d t \\
& \leq C_{q} \cdot C_{q, h} r^{-(q+1 / 2)} r^{-(q+2)} \leq C_{q} \cdot C_{q, h} r^{-(q+3 / 2)} .
\end{aligned}
$$

An application of Hölder's inequality together with (3) yields

$$
\begin{aligned}
\left|I_{2}\right| & \leq\left(\int_{|t|>r / 2}\left|(r-t)^{-q} \cdot J_{p}(r-t)\right|^{4} d t\right)^{1 / 4}\left(\int_{|t|>r / 2}|h(t)|^{4 / 3} d t\right)^{3 / 4} \\
& \leq C_{p}^{\prime} \cdot C_{p, h} \cdot\left(\int_{-\infty}^{\infty}\left|t^{-q} \cdot J_{p}(t)\right|^{4} d t\right)^{1 / 4} r^{-(q+3 / 2)}, \quad r>1 .
\end{aligned}
$$


For $p \geq q \geq 0$, the well-known fact that

$$
\lim _{t \rightarrow 0} J_{p}(t) / t^{p}<\infty \text { and } \varlimsup_{t \rightarrow \infty}\left|J_{p}(t) t^{1 / 2}\right|<\infty
$$

implies $\int_{\infty}^{\infty}\left|t^{-q} J_{p}(t)\right|^{4} d t<\infty$. Summarizing the above discussion, we get

$$
\left|I_{2}\right|+\left|I_{3}\right| \leq C(p, q, h) \cdot r^{-(q+3 / 2)} \quad \text { for } r>1 .
$$

Next recall the asymptotic formula $J_{p}(t)=(2 / \pi)^{1 / 2} t^{-1 / 2} \cos \left(t-a_{p}\right)+Q(t)$, where $|Q(t)| \leq C_{p} \cdot|t|^{-3 / 2}$ for all $t \in R$ and $C_{p}$ is a constant dependent on $p$ alone. Then, we have

$$
\left\{\begin{array}{l}
I_{1}=I_{1}^{\prime}+I_{2}^{\prime}+I_{3}^{\prime}, \\
I_{1}^{\prime}=\left(\frac{2}{\pi}\right)^{1 / 2} \int_{|t| \leq r / 2}\left[(r-t)^{-(q+1 / 2)}-r^{-(q+1 / 2)}\right] \cos \left(r-t-a_{p}\right) h(t) d t \\
I_{2}^{\prime}=\left(\frac{2}{\pi}\right)^{1 / 2} r^{-(q+1 / 2)} \cdot \int_{|t| \leq r / 2}\left[\cos \left(r-t-a_{p}\right)-\cos \left(r-a_{p}\right) \cos t\right] h(t) d t \\
I_{3}^{\prime}=\int_{|t| \leq r / 2}(r-t)^{-q} Q(r-t) h(t) d t .
\end{array}\right.
$$

But $|r-t| \geq r / 2$ for $|t| \leq r / 2$. Hence,

$$
\left|I_{3}^{\prime}\right| \leq C_{p}\left(\frac{1}{2} r\right)^{-(q+3 / 2)} \int_{-\infty}^{\infty}|h(t)| d t .
$$

Note that $h$ is even. A direct calculation shows

$$
I_{2}^{\prime}=-\left(\frac{2}{\pi}\right)^{1 / 2} \sin \left(r-a_{p}\right) r^{-(q+1 / 2)} \int_{|t| \leq r / 2} h(t) \sin t d t=0 .
$$

Furthermore, it is easy to check that for $|t| \leq \frac{1}{2} r$,

$$
\left|\left(1-\frac{t}{r}\right)^{-(q+1 / 2)}-1\right| \leq\left(q+\frac{1}{2}\right) q^{(q+1 / 2)} \cdot \frac{|t|}{r} .
$$

This fact yields

$$
\begin{aligned}
\left|I_{1}^{\prime}\right| & \leq\left(\frac{2}{\pi}\right)^{1 / 2} \cdot r^{-(q+1 / 2)} \int_{|t| \leq r / 2}\left|\left(1-\frac{t}{r}\right)^{-(q+1 / 2)}-1\right| \cdot|h(t)| d t \\
& \leq\left(q+\frac{1}{2}\right) 2^{(q+1 / 2)} \cdot r^{-(q+3 / 2)} \int_{-\infty}^{\infty}|\operatorname{th}(t)| d t .
\end{aligned}
$$

From (8)-(11), we get

$$
\left|I_{1}\right| \leq \tilde{C}(p, q, h) r^{-(q+3 / 2)} \quad \text { for } r>0 .
$$

Lemma 2 follows from (4), (7), and (12) immediately.

Lemma 3. Let $h(t)$ be given as in Lemma 2. For $p \geq 0$, let $H(r)=$ $\left[\left(t^{-p} \cdot J_{p}(t)\right) * h(t)\right](r)$. Then

$$
\frac{d H(r)}{d r}=-\left(\frac{2}{\pi}\right)^{1 / 2} \cdot r^{-(p+1 / 2)} \cdot\left[\hat{h}(1) \sin \left(r-\frac{1}{2} \pi p-\frac{1}{4} \pi\right)+R_{2}(r)\right]
$$

and $\left|R_{2}(r)\right| \leq C_{2} \cdot r^{-1}$ for $r>1$, where $C_{2}$ is a constant independent of $r$. 
Proof. Note that

$$
\frac{d H(r)}{d r}=\left[\left(\frac{d}{d t}\left(t^{-p} \cdot J_{p}(t)\right)\right) * h(t)\right](r) \quad \text { and } \quad \frac{d}{d t}\left(t^{-p} \cdot J_{p}(t)\right)=-t^{-p} \cdot J_{p+1}(t) \text {. }
$$

Since $\cos \left(r-\frac{1}{2} \pi(p+1)-\frac{1}{4} \pi\right)=\sin \left(r-\frac{1}{2} \pi p-\frac{1}{4} \pi\right)$, Lemma 3 is a direct consequence of Lemma 2.

Remark 2. The basic knowledge about the Bessel function used in the proofs of Lemmas 2 and 3 can be found in Watson's book [3].

\section{Proof of THE THEOREM}

Let $H(\theta, r)=\left[\left(t^{-(n-2) / 2} J_{(n-2) / 2}(t)\right) * \hat{g}(\theta, t)\right](r)$, where $g$ is given as in Lemma 1. For each fixed $\theta$, since $g(\theta, s) \in C_{0}^{\infty}(R)$ is even (see Proposition 2), $\hat{g}(\theta, t) \in \mathscr{S}(R)$ is also even. Applying Lemmas 2 and 3 we get

$$
\begin{aligned}
H(\theta, r)= & \left(\frac{2}{\pi}\right)^{1 / 2} r^{-(n-1) / 2} \\
& \cdot\left[\hat{\hat{g}}(\theta, 1) \cos \left(r-\frac{1}{4} \pi(n-2)-\frac{1}{4} \pi\right)+R_{1}(\theta, r)\right] \\
= & \left(\frac{2}{\pi}\right)^{1 / 2} r^{-(n-1) / 2}\left[g(\theta,-1) \cos \left(r-\frac{1}{4} \pi n+\frac{1}{4} \pi\right)+R_{1}(\theta, r)\right]
\end{aligned}
$$

and

$$
\begin{aligned}
\frac{\partial H(\theta, r)}{\partial r}= & -\left(\frac{2}{\pi}\right)^{1 / 2} r^{-(n-1) / 2} \\
& \cdot\left[g(\theta,-1) \sin \left(r-\frac{1}{4} \pi n+\frac{1}{4} \pi\right)+R_{2}(\theta, r)\right] .
\end{aligned}
$$

Moreover, there exist constants $C_{1}(\theta)$ and $C_{2}(\theta)$ independent of $r$ such that $\left|R_{1}(\theta, r)\right| \leq C_{1}(\theta) r^{-1}$ and $\left|R_{2}(\theta, r)\right| \leq C_{2}(\theta) r^{-1}$ for $r>1$.

Proposition 3. There exists a constant $C_{0}$ independent of $\theta$ such that $\left|C_{1}(\theta)\right| \leq$ $C_{0}$ and $\left|C_{2}(\theta)\right| \leq C_{0}$ for all $\theta \in S^{n-1}$.

Proof. We easily see from the proof of Lemma 2 that, besides the dimension $n, C_{1}(\theta)$ and $C_{2}(\theta)$ depend only on

$$
\operatorname{Sup}_{t \in R}|\hat{g}(\theta, t)| \cdot|t|^{(n / 2+2)}, \quad \int_{-\infty}^{\infty}|\hat{g}(\theta, t)| d t, \quad \int_{-\infty}^{\infty}|t \hat{g}(\theta, t)| d t .
$$

Recall that, for any fixed $\theta, \hat{g}(\theta, t)$ is the Fourier transform of $g(\theta, s)$ whose support lies in an interval independent of $\theta$ (see Proposition 2). This fact implies that the first term in (15) is uniformly bounded about $\theta$, and hence the two integrals in (15) are also uniformly bounded. The proof of Proposition 3 is complete.

On the other hand, $g(\theta,-1) \neq 0$ (see Proposition 1) and $g(\theta,-1) \in$ $C^{\infty}\left(S^{n-1}\right)$ implies $\operatorname{Min}(|g(\theta,-1)|) \neq 0$. This fact, together with Proposition 3 combining (13) and (14), shows that there exists an absolute constant $R_{0}>0$ such that for any fixed $\theta \in S^{n-1}$,

$$
\left\{r>R_{0} ; H(\theta, r)=0\right\}=\left\{r_{j}(\theta) ; j=1,2, \ldots\right\},
$$


where $r_{j_{1}}(\theta) \neq r_{j_{2}}(\theta) \quad\left(j_{1} \neq j_{2}\right)$ and

$$
\left.\frac{\partial H(\theta, r)}{\partial r}\right|_{r=r_{j}(\theta)} \neq 0, \quad j=1,2, \ldots
$$

Apparently, $H(\theta, r) \in C^{\infty}\left(S^{n-1} \times R\right)$. Then the implicit function theorem asserts that $r_{j}(\theta) \in C^{\infty}\left(S^{n-1}\right)$.

Let $R_{0}^{\prime}=R_{0} / \operatorname{Min}(d(\theta))$. According to Lemma 1 and the above discussion, we know that

$$
N(S, d u) \cap\left\{y \in R^{n} ;|y|>R_{0}^{\prime}\right\} \subseteq \bigcup_{j=1}^{\infty}\left\{\frac{r_{j}(\theta)}{d(\theta)} \theta ; \theta \in S^{n-1}\right\} .
$$

Obviously, $r_{j}(\theta) \theta / d(\theta) \quad\left(\theta \in S^{n-1}\right)$ is a hypersurface diffeomorphic to $S^{n-1}$. We conclude the proof of the theorem by mentioning that $N(S, d u) \cap\{y \in$ $\left.R^{n} ;|y| \leq R_{0}^{\prime}\right\}$ is a compact set.

\section{ACKNOWLEDGMENT}

The author would like to thank the referee for his corrections and comments that led to the result in this note.

\section{REFERENCES}

1. A. Greenleaf, Principal curvature and harmonic analysis, Indiana Univ. Math. J. 30 (1981), 519-536.

2. E. M. Stein, Problems in harmonic analysis related to curvature and osillatory integrals, Proc. Internat. Congr. Math., Berkeley, CA, 1986, pp. 196-221.

3. G. N. Watson, A treatise on the theory of Bessel function, Cambridge Univ. Press, Cambridge, 1922.

Department of Mathematics, Hangzhou University, Hangzhou, Zhejiang, 310028 , PEOPle's RePublic of China 